\title{
FITOTECNIA
}

\section{RENDIMENTO DE GRÃOS E QUALIDADE TECNOLÓGICA DE GENÓTIPOS DE TRIGO EM TRÊS ZONAS TRITÍCOLAS DO ESTADO DE SÃO PAULO NO BIÊNIO 1994-95 ${ }^{(1)}$}

\author{
JOÃO CARLOS FELICIO ${ }^{(2)}$; CARLOS EDUARDO DE OLIVEIRA \\ CAMARGO $^{(2)}$; ROGERIO GERMANI ${ }^{(3)}$; JOSÉ GUILHERME DE FREITAS ${ }^{(2)}$; \\ ANTONIO WILSON PENTEADO FERREIRA-FILHO ${ }^{(2)}$
}

\begin{abstract}
RESUMO
\end{abstract}
Foram avaliados o rendimento de grãos, a estabilidade e adaptabilidade ambiental, a tolerância ao alumínio tóxico e a qualidade tecnológica da farinha de vinte genótipos de trigo em três regiões tritícolas do Estado de São Paulo (Zonas A e B, sequeiro; Zona H, com irrigação por aspersão), no biênio 1994-95. Os resultados mostraram que: (a) os genótipos tiveram comportamento regional diferenciado quanto ao rendimento de grãos; (b) os genótipos IAC 289, IAC 340 e BR 18 mostraram-se, em média os mais produtivos, não diferindo do 'IAC 304', 'OCEPAR 14', 'IAC 350', 'Panda', 'IAC 60' e 'IAC 348'; (c) os genótipos apresentaram, em geral, boa estabilidade produtiva nas diferentes condições ambientais; (d) os genótipos IAC 289, IAC 340 e IAC 304 foram responsivos à melhoria ambiental e 'IAC 227', adaptado a ambientes desfavoráveis; (e) os genótipos IAC 24, IAC 120, IAC 227, OCEPAR 14, IAC 348, Panda, BR 18, IAC 319, IAC 346, IAPAR 60, IAC 332 e IAC 347 foram tolerantes à toxicidade de $\mathrm{Al}^{3+} ;(\mathrm{f})$ a farinha dos diferentes genótipos apresentou baixa atividade amilásica; $(\mathrm{g})$ os genótipos IAC 340, IAC 227, IAC 319 e IAC 60 propiciaram farinha com baixos valores de W (energia de deformação da massa) e baixa estabilidade, características próprias de farinha de glúten fraco. Os demais genótipos revelaram característica de glúten forte.

Palavras-chave: Triticum aestivum L., rendimento de grãos, estabilidade, adaptabilidade, tolerância a $\mathrm{Al}^{3+}$, características tecnológicas da farinha.

\section{ABSTRACT \\ GRAIN YIELD AND TECHNOLOGICAL QUALITY OF WHEAT GENOTYPES IN THE STATE OF SÃO PAULO, BRAZIL, DURING 1994-95}

Twenty wheat genotypes were evaluated in three different wheat regions in the State of São Paulo, Brazil (Zones A and B - upland conditions, and Zone H - sprinkler irrigation) during the 1994-95 season. Grain yield stability and adaptability, tolerance to aluminum toxicity and flour quality parameters were determined. The results showed: a) the genotypes exhibited different regional behavior in relation to grain yield; b) the genotypes IAC 289, IAC 340 and BR 18 displayed the highest grain yields, considering all experiments, and they did not differ from 'IAC 304', 'OCEPAR 14', 'IAC $350^{\prime}$,'Panda', 'IAC $60^{\prime}$ and 'IAC $348^{\prime}$; c) the majority of the genotypes showed no significant regression deviation $\left(S^{2} d\right)$ values, therefore they did not display diversity when grouped; d) 'IAC 289', 'IAC 340' and 'IAC 304' were responsive to favorable environments $(\mathrm{b}>1)$ and 'IAC 227 ' was responsive to unfavorable environments ( $b$ < ); e) the genotypes IAC 24, IAC 120, IAC 227, OCEPAR 14, IAC 348, Panda, BR 18, IAC 319, IAC 346, IAPAR 60, IAC 332 and IAC 347 were considered tolerant to $\mathrm{Al}^{3+}$

(1) Recebido para publicação em 1. ${ }^{1}$ de abril de 1998 e aceito em 20 de janeiro de 2000.

$\left({ }^{2}\right)$ Centro de Plantas Graníferas, Instituto Agronômico (IAC), Caixa Postal 28, 13001-970 Campinas (SP).

(3) EMBRAPA, Centro Nacional de Pesquisa de Tecnologia Agroindustrial de Alimentos (CTAA) 23020-470 Rio de Janeiro (RJ). 
toxicity; f) the flour of the genotypes showed low amylase activity; g) the flour of the genotypes IAC 340, IAC 227, IAC 319 and IAC 60 had low W (dough deformation energy) values and low stability, characteristics of weak gluten flour. The other genotypes showed strong gluten characteristics.

Key words: Triticum aestivum L., wheat, grain yield, adaptability, tolerance to $\mathrm{Al}^{3+}$, flour quality.

\section{INTRODUÇÃO}

O clima é uma das principais causas das grandes variações de rendimento na produção de trigo, entretanto há muitas maneiras de minimizar seus efeitos. Na coleção mundial de germoplasma de trigo encontram-se variedades que diferem quanto à tolerância ao frio, às geadas durante o estádio de plântula, ao calor, aos ventos quentes e secos e aos períodos de estiagem (de diferentes durações) nas diversas fases do desenvolvimento da planta, que podem atenuar esses problemas (HANsON et al., 1982).

Tal capacidade de alguns genótipos de apresentarem excelente adaptabilidade e estabilidade em diferentes ambientes tem sido apreciada pelos geneticistas e fitomelhoristas (CARVALHO, 1982).

As principais áreas onde se cultiva o trigo, no mundo, estão concentradas entre os paralelos de 30 e 55 graus de latitude, em ambos os hemisférios, em climas moderadamente seco ou moderadamente úmido e temperado. Agronomicamente, o clima favorável para o trigo deve apresentar inverno suave, verão quente com alta radiação solar, sem chuvas fortes e suprimento de água fornecido pela umidade armazenada no solo (MотA, 1982).

As condições de clima e solo das regiões onde tradicionalmente se produz o trigo, no Brasil, são bastante diferentes das observadas em outros países. Esse fato tem sido constantemente negligenciado nas análises dos problemas da triticultura nacional, principalmente quando são utilizadas comparações internacionais como argumento analítico (DUARTE, 1974).

KALCKMANN et al. (1965), considerando as conclusões de experimentos conduzidos em condição de sequeiro por Bittencourt, no período de 1929 a 1946, em Campinas (SP) - portanto fora da faixa de recomendação para a cultura do trigo - verificaram que os problemas relativos ao trigo no Estado de São Paulo, advindos da distribuição irregular das chuvas por ocasião da semeadura, perfilhamento e florescimento, seriam solucionados com o emprego de irrigação.

Em regiões onde o trigo amadurece durante o período das chuvas, algumas variedades podem apresentar germinação dos grãos nas espigas antes da operação de colheita, diminuindo a qualidade de panificação do cereal (HANsON et al. 1982).

A qualidade industrial do trigo depende, principalmente, das proteínas que estão intimamente li- gadas ao patrimônio genético de uma variedade, as quais sofrem alterações causadas pelos fatores ambientais. Com relação às características de qualidade, há cultivares que interagem melhor com o ambiente em comparação a outros. Os experimentos de avaliação de cultivares em diferentes locais, com técnicas de cultivo definidas, fornecem informações importantes nesse aspecto (MANDARINO, 1993).

O objetivo do presente trabalho foi avaliar o comportamento agronômico e a qualidade industrial para panificação - de genótipos de trigo cultivados em diferentes regiões climáticas do Estado de São Paulo.

\section{MATERIAL E MÉTODOS}

Visando avaliar o comportamento e a qualidade industrial de genótipos de trigo para a panificação nas condições de sequeiro e de irrigação por aspersão - instalaram-se experimentos nas seguintes zonas tritícolas do Estado de São Paulo, no período de 1994 a 1995: zona A em Maracaí; zona B em Itaberá; e zona $\mathrm{H}$ em Campinas. Para a instalação dos experimentos foram seguidas as recomendações da Comissão Técnica de Trigo da Secretaria de Agricultura e do Abastecimento do Estado de São Paulo (Instituto Agronômico, Campinas, 1996).

Os experimentos foram delineados em blocos ao acaso com vinte genótipos (tratamentos) relacionados no quadro 1 e quatro repetições por local. Cada parcela constou de seis linhas de $3 \mathrm{~m}$ de comprimento com espaçamento de $0,20 \mathrm{~m}$ e separação lateral de $0,60 \mathrm{~m}$ entre as parcelas. Procedeu-se à semeadura utilizando-se 80 sementes viáveis por metro de sulco, tendo sido, no devido tempo, efetuada a colheita da área total das parcelas.

A adubação mineral foi feita a lanço antes da semeadura e, posteriormente, incorporada ao solo. As quantidades de fertilizantes aplicadas nos diferentes locais foram definidas de acordo com as recomendações de adubação e calagem para o Estado de São Paulo (RAIJ et al., 1996).

Nos experimentos irrigados adotou-se o método proposto por Silva et al. (1984), o qual consiste em uma irrigação de $40 \mathrm{~mm}$ a $60 \mathrm{~mm}$ após a semeadura, com a finalidade de umedecer o solo. As irrigações complementares foram realizadas quando a média das leituras dos tensiômetros (instalados em diversos pontos à profundidade de $12 \mathrm{~cm}$ ) indicava $-0,6 \mathrm{MPa}$; 
determinou-se a lâmina líquida aplicada (pela evaporação acumulada) medida no tanque classe $\mathrm{A}$, entre os intervalos das irrigações.

A avaliação do rendimento de grãos foi realizada determinando-se, em gramas, a produção total de cada parcela extrapolada para quilogramas por hectare.

Os genótipos estudados foram também testados em soluções nutritivas contendo 0, 2, 6 e $10 \mathrm{mg} \mathrm{Al} / \mathrm{L}$, conforme Moore et al.(1976), CAMARgo e Oliveira (1981) e CAMARGO et al.(1987).

Para a avaliação da qualidade tecnológica dos genótipos, foram utilizados individualmente os experimentos semeados nas diferentes zonas tritícolas durante o biênio 1994-95. As produções de grãos das quatro parcelas de cada experimento (local) foram reunidas para se obter quantidade suficiente de grãos para as determinações a seguir relacionadas:

Teor de água dos grãos: determinado pelo método n. ${ }^{\circ} 44-15 A$ da AACC (1983).

Peso hectolítrico(PH): determinado utilizando-se a balança Dalle Molle, considerando-se a massa (em quilograma) de $1 \mathrm{~L}$ de grãos, obtida após fazer fluir livremente uma amostra contida em cone de alimen- tação, em um recipiente de coleta de volume de $1 \mathrm{~L}$ (BRASIL, 1999).

Massa de mil sementes (mms): determinada em balança correspondendo à massa (em gramas) de mil sementes tomadas ao acaso da produção total de cada parcela.

Rendimento de extração de farinha (moagem): determinado utilizando-se moinho experimental $\mathrm{Bra}$ bender Quadrumar Senior trabalhando com $2 \mathrm{~kg}$ de grãos, com base no método da AACC n. ${ }^{\circ} 26-20$ (1983). Entre 16 e 20 horas antes da moagem os grãos tiveram seu teor de água ajustado para $15 \%$.

Dureza do grão (DZ): determinada pelo tempo de moagem de $4 \mathrm{~g}$ de amostra - quanto menor a dureza, maior o tempo de moagem - utilizando-se o Brabender Micro-hardness Teste (Brabender OHG, Dvisburg).

Número de queda (Falling Number): determinado pelo método $\mathrm{n} .{ }^{\circ}$ 56-81B da AACC (1983).

Propriedades de mistura da massa: determinadas no farinógrafo Brabender, pelo sistema farinha-água, de acordo com o método n. ${ }^{\circ}$ 54-21 da AACC (1983). Os parâmetros usados para interpretar o farinógrafo foram: absorção de água (\%), tempo de desenvolvimento da massa (TDM) (min), estabilidade (EST)

Quadro 1. Genótipos de Triticum aestivum L. e respectivos cruzamentos avaliados nos experimentos semeados em três diferentes regiões tritícolas do Estado de São Paulo no biênio 1994-95

\begin{tabular}{|c|c|}
\hline Genótipo & Cruzamento \\
\hline Anahuac & II12300/LR64/8156/3/Nortenõ 67 \\
\hline BR 18 & D6301/NAI60//WQ/RM/3/CNO*2/CHR \\
\hline IAC 24 & IAS51/4/SON64/Y50E//GTO/2*CIANO \\
\hline IAC 60 & IRN 33-70/MARINGÁ \\
\hline IAC 120 & IRN 33-70/MARINGÁ \\
\hline IAC 227 & CNT 9/BH 1146 \\
\hline IAC 287 & $\mathrm{HIM} / \mathrm{COC} / \mathrm{NAC}$ \\
\hline IAC 289 & KVZ/BUHO"S"//KAL/BLUEBIRD \\
\hline IAC 304 & MIR 808/BOW \\
\hline IAC 319 & ALD/BH 1146/ /LONDRINA \\
\hline IAC 332 & EMU"S" /IAC 67 \\
\hline IAC 340 & BH 1146/7C/IAC 60 \\
\hline IAC 346 & PF70354/ALD"S"//YACO"S"/3/IAC 73 \\
\hline IAC 347 & PF70354/MUS//JUP/MEU/3/GJO/4/IAC 73 \\
\hline IAC 348 & $\mathrm{TP} / / \mathrm{CNO} 67 / \mathrm{NO} / 3 / \mathrm{BB} / \mathrm{CNO} / 4 / \mathrm{ZA75} / 5 / \mathrm{TTM} / 6 / \mathrm{IAC} 60$ \\
\hline IAC 350 & 2109.36/SERI \\
\hline IAPAR 28 & KVZ/BUHO"S"//KAL/BLUEBIRD \\
\hline IAPAR 60 & BJY"S'/JUP73//TAN"S" \\
\hline OCEPAR 14 & IAS64/ALDAN/6/COC/5/PICHON/4/KT54/N10B//KT54B/3/NAR \\
\hline Panda & IDS1528/S45//PARG281 \\
\hline
\end{tabular}


(min), índice de tolerância à mistura (ITM) e o valor volumétrico (VV), em unidades farinográficas.

Propriedades de extensão da massa: determinadas no alveógrafo Chopin, de acordo com o método da AACC n. ${ }^{\circ}$ 54-30 (1983). Os parâmetros básicos das curvas alveográficas estudados foram: pressão máxima (P), abscissa média de ruptura (L), índice de configuração da curva (P/L) e a energia de deformação da massa $(W)$.

O rendimento de grãos de cada experimento foi submetido à análise de variância individual (local) no biênio 1994-95. Para a avaliação das interações anos vs. genótipos foram feitas análises conjuntas dos experimentos instalados no mesmo local, nos dois anos considerados. Foi realizada também a análise de variância conjunta para os experimentos em condições de sequeiro (Itaberá e Maracaí) e para o conjunto dos experimentos (sequeiro e irrigado), visando averiguar a ocorrência das seguintes interações: locais vs. genótipos, anos vs. genótipos, anos vs. locais e anos vs. locais vs. genótipos. Utilizou-se para a comparação das médias o teste de Duncan, de acordo com Pimentel-Gomes (1970).

Foram determinadas também a estabilidade e a adaptabilidade através do método proposto por EBERHART e RUSSELL (1966), com base nos coeficientes de cada genótipo em relação ao índice ambiental, considerando como genótipo ideal aquele que apresenta alto rendimento médio de grãos, coeficiente de regressão igual a 1,0 (b=1) e desvios da regressão igual a zero $\left(S^{2} \mathrm{~d}=0\right)$.

Os dados médios anuais de peso hectolítrico, massa de mil sementes, dureza e extração experimental de farinha, foram submetidos à análise de variância.

\section{RESULTADOS E DISCUSSÃO}

As médias das precipitações pluviométricas representadas na figura 1 (biênio 1994-95), relativas às localidades de Pedrinhas Paulista (Zona A), Itararé (Zona B) e Campinas (Zona $\mathrm{H}$ ), são representativas das referidas zonas tritícolas no Estado de São Paulo. Verificou-se nas três regiões durante o período do estabelecimento inicial da cultura do trigo (abril a maio), ausência de deficiência hídrica acentuada, mesmo apresentando períodos relativamente longos sem chuvas. Nos meses de julho e agosto houve deficiência hídrica acentuada, favorecendo o amadurecimento e a colheita do cereal em condições ótimas. Nos meses de setembro e, principalmente outubro, as precipitações voltaram a ocorrer com maior intensidade. Colheitas nesse período poderiam prejudicar a qualidade dos grãos, principalmente se ocorresse germinação dos grãos na espiga. Na zona $\mathrm{H}$, por apre-
Zona A - Pedrinhas Paulista

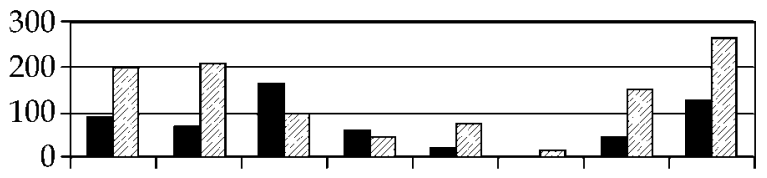

Zona B - Itararé

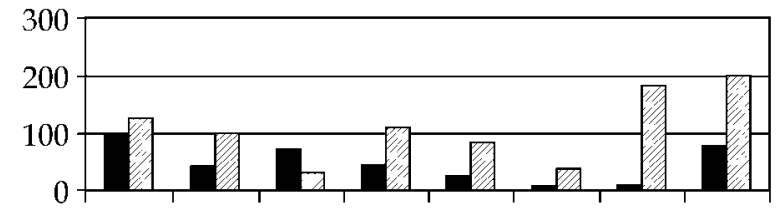

Zona H - Campinas

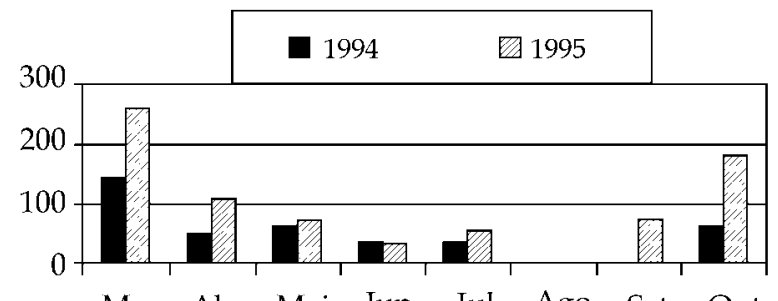

Mar. Abr. Mai. Jun. Jul. Ago. Set. Out.

Meses

Figura 1. Distribuição pluvial (totais mensais) referentes ao biênio 1994-95 para as localidades de Pedrinhas Paulista (Zona A), Itararé (Zona B) e Campinas (Zona H) no Estado de São Paulo.

sentar menor índice de precipitação pluvial, o emprego da irrigação tornou-se necessário para a obtenção de bom rendimento de grãos.

Encontram-se no quadro 2: a produção de grãos dos genótipos - avaliados em condição de sequeiro nas localidades de Maracaí e Itaberá e com irrigação por aspersão em Campinas - bem como os resumos da análise de variância dos experimentos isolados e em conjunto, para o biênio 1994-95.

A análise conjunta para rendimento de grãos entre todos os experimentos apresentou efeitos significativos para anos, locais (condição de cultivo de sequeiro ou irrigado) e genótipos. As interações anos vs. locais, locais vs. genótipos e anos vs. locais vs. genótipos foram significativas, exceto a interação anos vs. genótipos.

Para as condições de sequeiro a produtividade média, em 1994, foi de 3.323 kg.ha ${ }^{-1}$ e de 3.750 kg.ha ${ }^{-1}$ em 1995. O alto índice pluvial de 1995 contribuiu para o aumento do rendimento de grãos. A Zona $\mathrm{H}$, com irrigação por aspersão, apresentou rendimento médio de 4.575 kg.ha ${ }^{-1}$ em 1994 e de 3.330 kg.ha ${ }^{-1}$ em 1995.

A análise conjunta, considerando-se os experimentos de sequeiro (Zonas A e B), apresentou efeitos significativos para todas as causas de variação. Nos 
Quadro 2. Produtividade média de grãos dos genótipos de trigo avaliados em condição de sequeiro nas localidades de Itaberá (Zona B) e Maracaí (Zona A) e sob irrigação por aspersão em Campinas (Zona H) com o resumo da análise de variância conjunta para as condições de sequeiro e a conjunta geral, para o biênio 1994-95 no Estado de São Paulo

\begin{tabular}{|c|c|c|c|c|c|}
\hline Genótipo & Itaberá & Macaraí & Média & Campinas & Média geral \\
\hline & & 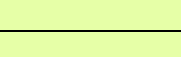 & kg.ha ${ }^{-1}$ & & \\
\hline IAC 289 & $5.835 a$ & 2.283 & $4.059 \mathrm{a}$ & 3.831ce & $3.983 a$ \\
\hline IAC 340 & 5.451ac & 2.023 & $3.737 \mathrm{~b}$ & $4.437 \mathrm{ab}$ & $3.971 \mathrm{a}$ \\
\hline BR 18 & $5.082 \mathrm{cf}$ & 2.245 & $3.663 \mathrm{bd}$ & $4.547 \mathrm{a}$ & $3.958 a$ \\
\hline IAC 304 & $5.600 \mathrm{ab}$ & 1.947 & $3.773 b$ & $4.253 \mathrm{ac}$ & $3.933 \mathrm{ab}$ \\
\hline OCEPAR 14 & $5.177 \mathrm{be}$ & 2.248 & $3.712 \mathrm{bc}$ & $4.237 \mathrm{ac}$ & $3.887 \mathrm{ac}$ \\
\hline IAC 350 & $5.105 \mathrm{cf}$ & 2.343 & $3.724 b$ & $4.085 \mathrm{ad}$ & 3.844ad \\
\hline Panda & $5.109 \mathrm{cf}$ & 1.918 & $3.514 \mathrm{bd}$ & $4.393 \mathrm{ab}$ & 3.807ae \\
\hline IAC 60 & $4.874 \mathrm{eg}$ & 2.034 & $3.454 \mathrm{be}$ & $4.377 \mathrm{ab}$ & 3.762ae \\
\hline IAC 348 & 4.767eg & 2.374 & $3.571 \mathrm{bd}$ & 4.131ad & 3.757ae \\
\hline IAPAR 60 & $5.103 \mathrm{cf}$ & 2.141 & $3.622 \mathrm{bd}$ & $3.766 \mathrm{df}$ & $3.670 \mathrm{be}$ \\
\hline IAC 347 & $4.919 \mathrm{dg}$ & 2.013 & $3.466 \mathrm{bd}$ & $3.996 \mathrm{bd}$ & $3.643 \mathrm{cf}$ \\
\hline IA332 & $4.694 \mathrm{fg}$ & 2.074 & 3.584ce & $4.118 \mathrm{ad}$ & $3.628 \mathrm{cf}$ \\
\hline IAC 319 & $4.887 \mathrm{dg}$ & 2.211 & $3.549 \mathrm{bd}$ & $3.739 \mathrm{df}$ & $3.613 \mathrm{df}$ \\
\hline IAC 346 & $4.849 \mathrm{eg}$ & 2.077 & $3.463 \mathrm{bd}$ & 3.818ce & $3.582 \mathrm{df}$ \\
\hline IAC 287 & $4.765 \mathrm{eg}$ & 2.252 & $3.508 \mathrm{bd}$ & $3.716 \mathrm{df}$ & $3.578 \mathrm{df}$ \\
\hline IAPAR 28 & $5.346 \mathrm{bd}$ & 1.979 & $3.662 \mathrm{bd}$ & $3.358 \mathrm{fg}$ & 3.561ef \\
\hline IAC 120 & $4.521 \mathrm{~g}$ & 2.230 & 3.375 & 3.863ce & $3.538 \mathrm{ef}$ \\
\hline IAC 227 & $4.472 \mathrm{~g}$ & 2.242 & 3.357de & $3.482 \mathrm{eg}$ & $3.399 \mathrm{fg}$ \\
\hline IAC 24 & $3.919 \mathrm{~h}$ & 2.056 & $2.988 \mathrm{f}$ & $3.658 \mathrm{dg}$ & $3.211 \mathrm{~g}$ \\
\hline Anahuac & $4.476 \mathrm{~g}$ & 1.817 & $3.146 \mathrm{ef}$ & $3.245 \mathrm{~g}$ & $3.179 \mathrm{~g}$ \\
\hline Média & $4.948 \mathrm{~A}$ & $2.125 B$ & & 3.953 & \\
\hline F Ano & $161,75^{*}$ & $4,25^{*}$ & $120,6^{*}$ & $283,77^{*}$ & $30,22^{*}$ \\
\hline F Local & & & $4.911,1^{*}$ & & $5.99,74^{*}$ \\
\hline F Genótipo & $12,28^{*}$ & $0,92 \mathrm{~ns}$ & $5,39 *$ & $4,90^{*}$ & $7,74^{*}$ \\
\hline F Ano vs. Local & & & $48,58^{*}$ & & $73,96^{*}$ \\
\hline F Ano vs. Genótipo & $5,22^{*}$ & $0,94 \mathrm{~ns}$ & $2,66^{*}$ & $4,48^{*}$ & $1,32 \mathrm{~ns}$ \\
\hline F Local vs. Genótipo & & & $5,22^{*}$ & & $4,41^{*}$ \\
\hline F Ano vs. Local vs. Genótipo & & & $2,52^{*}$ & & $4,74^{*}$ \\
\hline $\mathrm{CV}(\%)$ ano & & & 1,55 & 0,94 & 0,91 \\
\hline CV(\%) local & & & 2,27 & & 4,49 \\
\hline $\mathrm{CV}(\%)$ genótipos & 7,10 & 21,04 & 11,33 & 11,82 & 11,13 \\
\hline
\end{tabular}

Médias seguidas por letras distintas diferem entre si pelo teste de Duncan a $5 \%$; ${ }^{*}=$ significativo a $5 \%$; ns não significativo pelo teste F.

experimentos de Itaberá (Zona B) os efeitos foram significativos para ano, genótipos e ano vs. genótipos, com produtividade média de grãos de $4.948 \mathrm{~kg} \cdot \mathrm{ha}^{-1}$, superior à obtida em Maracaí (Zona A), de 2.125 hg.ha ${ }^{-1}$. Neste último local os efeitos foram significativos somente entre os anos. A Zona B apresentou chuvas melhor distribuídas durante o ciclo da cultura. Na zona $\mathrm{H}$, com irrigação por aspersão, a produtividade média foi de $3.953 \mathrm{~kg} \cdot \mathrm{ha}^{-1}$, também apresentando efeitos significativos para ano, genótipos e para a interação anos vs. genótipos.

Comparando-se a produção de grãos dos genótipos pelo teste de Duncan ao nível de 5\% (na média geral das três regiões), destacaram-se os seguintes: IAC 289, IAC 340 e BR 18, não diferindo estatisticamente dos genótipos IAC 304, OCEPAR 14, IAC 350, Panda, IAC 60 e IAC 348. Na zona A, 'IAC 348' e 'IAC 350' apresentaram tendência a serem mais 
produtivos, embora não diferindo estatisticamente dos demais genótipos. Na zona B, 'IAC 289' foi o mais produtivo com $5.835 \mathrm{~kg}$.ha ${ }^{-1}$ no biênio, não se diferenciando dos genótipos IAC 340 e IAC 304. Com irrigação por aspersão, na zona $\mathrm{H}$, destacaram-se quanto ao rendimento de grãos os genótipos BR 18, IAC 340, IAC 304, OCEPAR 14, IAC 350, Panda, IAC 60, IAC 348 e IAC 332. Os genótipos IAC 24 e Anahuac apresentaram baixa produtividade de grãos em ambas as condições de cultivo.

$\mathrm{Na}$ análise conjunta da estabilidade para rendimento de grãos nas três diferentes zonas tritícolas paulistas, a maioria dos genótipos apresentou desvio de regressão $\left(S^{2} d\right)$ não significativo ou variância negativa do erro, não indicando, portanto, diversidade no comportamento dos genótipos nos três locais considerados. Dessa forma, os genótipos apresentaram estabilidade, com exceção de 'IAC 289', 'IAPAR 28' e 'IAC 60 ', os quais se apresentaram instáveis quanto ao rendimento de grãos (Quadro 3). Em locais de condições climáticas favoráveis como Itaberá (zona B) e Campinas (zona $\mathrm{H}$ ) - onde se procedeu à irrigação - estes genótipos foram responsáveis por picos de altos rendimentos, ocasionando desvios de regressão significativos. As condições climáticas de Maracaí (zona A) foram menos favoráveis no decorrer desse período em relação às precipitações pluviais (Figura 1).

Os genótipos IAC 289, IAC 340 e IAC 304, considerando-se os valores significativos do coeficiente de regressão, tendem a apresentar comportamento melhor em ambientes favoráveis $(b>1)$, ao passo que 'IAC 227' apresenta adaptação a ambientes desfavoráveis $(b<1)$. Os genótipos IAC 289 e IAPAR 28 apresentaram desvio significativo da regressão $\left(\mathrm{S}^{2} \mathrm{~d}\right)$ a $1 \%$ e IAC 60 , a $5 \%$.

Quanto aos efeitos da solução de $\mathrm{Al}$ nos diversos genótipos de trigo, na concentração de $2 \mathrm{mg} \cdot \mathrm{L}^{-1} \mathrm{de} \mathrm{Al}^{3+}$, verificou-se que 'Anahuac', 'IAC 340', 'IAC 287' e 'IAC 304' foram sensíveis a essa concentração, sendo os demais genótipos tolerantes. Verificou-se o mesmo resultado quando foram adicionados $6 \mathrm{mg} . \mathrm{L}^{-1} \mathrm{de} \mathrm{Al}^{3+}$ na solução, ocasião em que também o genótipo IAPAR 28 mostrou-se sensível. Os genótipos IAC 24, IAC 120, IAC 227, OCEPAR 14, IAC 348, Panda, BR 18, IAC 319, IAC 346, IAPAR 60, IAC 332 e IAC 347, por outro lado, foram muito tolerantes à toxicidade de $\mathrm{Al}^{3+}$ por apresentarem crescimento das raízes na solução contendo $10 \mathrm{mg} . \mathrm{L}^{-1}$ de $\mathrm{Al}^{3+}$ (Quadro 4).

As diferenças ambientais entre áreas ou regiões distintas, incluindo as variações edafoclimáticas dentro de uma mesma área, podem influenciar na qualidade do trigo. As variações nos valores dos índices físicos, químicos e tecnológicos, bem como o teor e a
Quadro 3. Produtividade média de grãos, estimativa de parâmetros de estabilidade e adaptabilidade pelo método de EBERHART e RUSSELL (1966) e coeficientes de determinação $\left(\mathrm{R}^{2}\right)$ dos genótipos de trigo avaliados em condição de sequeiro nas localidades de Itaberá (Zona B) e Maracaí (Zona A) e com irrigação por aspersão em Campinas (Zona $H)$ no biênio 1994-95 no Estado de São Paulo

\begin{tabular}{|c|c|c|c|c|}
\hline Genótipo & $\begin{array}{r}\text { Produção } \\
\text { de grãos }\end{array}$ & $b^{(1)}$ & $S^{2} d^{(2)}$ & $\mathrm{R}^{2}$ \\
\hline & kg.ha ${ }^{-1}$ & & kg.ha ${ }^{-1}$ & $\%$ \\
\hline IAC 289 & 3983 & $1,20^{*}$ & $569^{* *}$ & 94,2 \\
\hline IAC 340 & 3971 & $1,22^{*}$ & - (3) & 99,6 \\
\hline BR 18 & 3958 & $1,03 \mathrm{~ns}$ & $314 \mathrm{~ns}$ & 96,9 \\
\hline IAC 304 & 3933 & $1,29^{* *}$ & - & 99,9 \\
\hline OCEPAR 14 & 3887 & $1,04 \mathrm{~ns}$ & - & 99,8 \\
\hline IAC 350 & 3844 & $0,97 \mathrm{~ns}$ & - & 99,9 \\
\hline PANDA & 3807 & $1,15 \mathrm{~ns}$ & $258 \mathrm{~ns}$ & 98,0 \\
\hline IAC 60 & 3762 & $1,04 \mathrm{~ns}$ & $350^{*}$ & 96,4 \\
\hline IAC 348 & 3757 & $0,86 \mathrm{~ns}$ & - & 99,0 \\
\hline IAPAR 60 & 3670 & $1,02 \mathrm{~ns}$ & $116 \mathrm{~ns}$ & 98,7 \\
\hline IAC 347 & 3643 & $1,03 \mathrm{~ns}$ & - & 99,8 \\
\hline IAC 332 & 3628 & $0,95 \mathrm{~ns}$ & $191 \mathrm{~ns}$ & 97,9 \\
\hline IAC 319 & 3613 & $0,93 \mathrm{~ns}$ & - & 99,2 \\
\hline IAC 346 & 3582 & $0,97 \mathrm{~ns}$ & - & 99,9 \\
\hline IAC 287 & 3578 & $0,87 \mathrm{~ns}$ & - & 99,4 \\
\hline IAPAR 28 & 3561 & $1,13 \mathrm{~ns}$ & $611^{* *}$ & 92,7 \\
\hline IAC 120 & 3538 & $0,82 \mathrm{~ns}$ & - & 99,4 \\
\hline IAC 227 & 3399 & $0,77^{*}$ & - & 98,9 \\
\hline IAC 24 & 3211 & $0,68 \mathrm{~ns}$ & $244 \mathrm{~ns}$ & 95,0 \\
\hline ANAHUAC & 3179 & $0,92 \mathrm{~ns}$ & $118 \mathrm{~ns}$ & 98,4 \\
\hline
\end{tabular}

(1) coeficiente de regressão linear; (2) variância do desvio de regressão; ${ }^{(3)}$ variância negativa do erro; ${ }^{*} \mathrm{e}^{* *}$ significativo a $5 \%$ e $1 \%$, pelos testes de $\mathrm{F}$ e $\mathrm{t} ; \mathrm{ns}=$ não-significativo.

qualidade das proteínas, são especialmente afetados pelas diferenças ambientais (MANDARINO, 1993).

Foram verificados, através da análise de variância dos dados relativos do peso hectolítrico (Quadro 5), efeitos significativos para local e para genótipo e nãosignificativos para ano. 'IAC 227' apresentou maior valor médio de $\mathrm{PH}$ no referido biênio $(82,41)$, superior ao obtido por 'IAC 120 ' (74,33), considerado o de menor valor. A análise de variância da massa de mil sementes (MMS), ao contrário do $\mathrm{PH}$, apresentou efeitos significativos entre os anos, locais e genótipos. O genótipo BR 18 obteve o maior valor $(42,70 \mathrm{~g})$ e o IAPAR 28 o menor valor $(30,72 \mathrm{~g})$. Tais parâmetros permitem evidenciar que - para cada genótipo - os 
Quadro 4. Comprimento médio das raízes dos genótipos de trigo em soluções-tratamento contendo diferentes concentrações de alumínio

\begin{tabular}{|c|c|c|c|c|}
\hline \multirow[t]{2}{*}{ Genótipo } & \multicolumn{4}{|c|}{ Concentração de alumínio (mg. $\left.\mathrm{L}^{-1}\right)$} \\
\hline & 0,0 & 2,0 & 6,0 & 10,0 \\
\hline \multirow[b]{2}{*}{ Anahuac } & & & & \\
\hline & 70,6 & 0,0 & 0,0 & 0,0 \\
\hline IAC 350 & 51,8 & 18,6 & 7,9 & 0,0 \\
\hline IAC 340 & 42,3 & 0,0 & 0,0 & 0,0 \\
\hline IAC 24 & 64,3 & 26,7 & 42,7 & 9,2 \\
\hline IAC 60 & 42,0 & 25,0 & 30,2 & 0,0 \\
\hline IAC 120 & 72,6 & 40,2 & 36,5 & 10,4 \\
\hline IAC 227 & 76,4 & 46,3 & 41,0 & 14,2 \\
\hline IAC 289 & 60,1 & 14,6 & 7,3 & 0,0 \\
\hline IAPAR 28 & 58,5 & 5,8 & 0,0 & 0,0 \\
\hline OCEPAR 14 & 77,8 & 32,6 & 16,8 & 3,7 \\
\hline IAC 287 & 75,0 & 0,0 & 0,0 & 0,0 \\
\hline IAC 348 & 55,4 & 33,5 & 45,7 & 6,6 \\
\hline Panda & 67,0 & 38,3 & 35,3 & 8,1 \\
\hline BR 18 & 39,2 & 36,1 & 31,4 & 4,3 \\
\hline IAC 319 & 56,3 & 38,3 & 43,0 & 12,7 \\
\hline IAC 346 & 50,8 & 32,7 & 8,9 & 1,8 \\
\hline IAPAR 60 & 67,2 & 32,2 & 21,1 & 3,5 \\
\hline IAC 332 & 65,0 & 30,5 & 32,7 & 3,6 \\
\hline IAC 304 & 59,1 & 0,0 & 0,0 & 0,0 \\
\hline IAC 347 & 50,8 & 33,3 & 28,4 & 14,2 \\
\hline
\end{tabular}

problemas ambientais ocorridos durante o processo de formação dos grãos, a ocorrência ou não de doenças fúngicas, bem como as práticas culturais utilizadas, podem afetar a qualidade dos mesmos (MANDARINO, 1993). Os dados de precipitação pluvial no biênio 1994-95 (de acordo com a Figura 1) evidenciaram condições distintas nas diferentes regiões tritícolas durante o desenvolvimento da cultura, capazes de influenciar na produtividade final de cada genótipo.

Os resultados dos testes de dureza dos grãos e de extração experimental de farinha (Quadro 5) foram expressos em porcentagem de extração (índice de rendimento); a análise de variância conjunta de tais dados demonstrou efeitos significativos entre os anos, locais e genótipos. Quanto à dureza dos grãos, os genótipos IAC 60, IAC 340, IAC 348, IAC 319, IAC 347, IAC 287, IAC 120 e IAC 227 foram considerados de textura mole e os outros genótipos, de textura dura. Nos genótipos de textura dura a extração de
Quadro 5. Resumo da análise conjunta de variância e a média das variáveis peso hectolítrico $(\mathrm{PH})$, massa de mil sementes (MMS), dureza (DZ) e extração experimental de farinha dos genótipos avaliados em condição de sequeiro em Itaberá e Maracaí e com irrigação por aspersão em Campinas, no biênio 1994-95

\begin{tabular}{lcccc}
\hline Genótipo & PH & MMS & DZ & Extração \\
\hline IAC 227 & kg.hl ${ }^{-1}$ & $\mathrm{~g}$ & $\mathrm{~s}$ & $\%$ \\
PANDA & $82,41 \mathrm{a}$ & $37,53 \mathrm{cg}$ & $53 \mathrm{df}$ & $66,97 \mathrm{gh}$ \\
BR 18 & $81,94 \mathrm{ab}$ & $34,04 \mathrm{gi}$ & $20 \mathrm{f}$ & $75,25 \mathrm{ab}$ \\
IAC 350 & $81,25 \mathrm{ad}$ & $38,67 \mathrm{be}$ & $21 \mathrm{f}$ & $75,50 \mathrm{ab}$ \\
ANAHUAC & $80,80 \mathrm{ae}$ & $34,15 \mathrm{gj}$ & $21 \mathrm{f}$ & $74,16 \mathrm{ac}$ \\
IAC 304 & $80,65 \mathrm{ae}$ & $39,39 \mathrm{bf}$ & $31 \mathrm{ef}$ & $73,11 \mathrm{bd}$ \\
IAC 319 & $80,54 \mathrm{ae}$ & $38,32 \mathrm{bf}$ & $101 \mathrm{bd}$ & $66,35 \mathrm{~h}$ \\
IAC 24 & $80,26 \mathrm{ae}$ & $34,85 \mathrm{fg}$ & $27 \mathrm{ef}$ & $71,51 \mathrm{ce}$ \\
OCEPAR 14 & $80,20 \mathrm{ae}$ & $40,64 \mathrm{ac}$ & $26 \mathrm{ef}$ & $73,72 \mathrm{ac}$ \\
IAC 332 & $80,08 \mathrm{ae}$ & $41,70 \mathrm{ab}$ & $21 \mathrm{f}$ & $76,42 \mathrm{a}$ \\
IAPAR 60 & $79,98 \mathrm{ae}$ & $32,89 \mathrm{hi}$ & $22 \mathrm{f}$ & $73,49 \mathrm{ad}$ \\
IAPAR 28 & $79,85 \mathrm{ae}$ & $30,72 \mathrm{i}$ & $22 \mathrm{f}$ & $73,78 \mathrm{ad}$ \\
IAC 289 & $79,58 \mathrm{be}$ & $35,48 \mathrm{eh}$ & $30 \mathrm{ef}$ & $73,84 \mathrm{ad}$ \\
IAC 340 & $79,28 \mathrm{ce}$ & $41,83 \mathrm{ab}$ & $143 \mathrm{~b}$ & $70,21 \mathrm{ef}$ \\
IAC 347 & $79,11 \mathrm{de}$ & $36,31 \mathrm{dh}$ & $88 \mathrm{be}$ & $71,13 \mathrm{de}$ \\
IAC 346 & $78,71 \mathrm{df}$ & $34,52 \mathrm{gh}$ & $30 \mathrm{ef}$ & $69,76 \mathrm{eg}$ \\
IAC 348 & $78,18 \mathrm{ef}$ & $37,01 \mathrm{cg}$ & $134 \mathrm{bc}$ & $67,65 \mathrm{fh}$ \\
IAC 287 & $78,10 \mathrm{ef}$ & $35,62 \mathrm{eh}$ & $88 \mathrm{be}$ & $65,62 \mathrm{~h}$ \\
IAC 60 & $76,50 \mathrm{fg}$ & $35,77 \mathrm{eh}$ & $197 \mathrm{a}$ & $67,63 \mathrm{fh}$ \\
IAC 120 & $74,33 \mathrm{~g}$ & $39,77 \mathrm{ad}$ & $79 \mathrm{cf}$ & $66,69 \mathrm{~h}$ \\
F Ano & $2,44 \mathrm{~ns}$ & $14,81^{*}$ & $4,16^{*}$ & $4,14^{*}$ \\
F Local & $51,71^{*}$ & $41,08^{*}$ & $3,46^{*}$ & $11,35^{*}$ \\
F Genótipo & $5,67^{*}$ & $7,71^{*}$ & $7,22^{*}$ & $15,04^{*}$ \\
\hline
\end{tabular}

* significativo a $5 \%$; ** significativo a $1 \%$; ns: não-significativo pelo teste $\mathrm{F}$.

farinha foi maior em relação ao de textura mole, excetuando-se 'IAC 340' e 'IAC 347' o qual apresentou $70,21 \%$ de extração.

O número de queda (Falling Number) (que caracteriza as farinhas de trigo quanto à atividade das amilases, permitindo prever o comportamento das mesmas durante a etapa de fermentação da massa no processo de panificação), bem como os testes realizados no alveógrafo de Chopin, são apresentados no quadro 6. Os dados do número de queda revelaram, em geral, baixa atividade amilásica (200-400 s), mostrando que a ocorrência de baixas precipitações pluviais por ocasião da colheita nos meses de agosto e início de setembro, nas zonas $\mathrm{A}, \mathrm{B}$ e $\mathrm{H}$, foram 
Quadro 6. Falling Number (número de queda) e parâmetros alveográficos médios para P/L e W ( por local) dos genótipos de trigo avaliados em condição de sequeiro em Itaberá e Maracaí e em Campinas com irrigação por aspersão, no biênio 1994-95

\begin{tabular}{|c|c|c|c|c|c|c|}
\hline \multirow{2}{*}{ Genótipo } & \multirow{2}{*}{$\mathrm{FN}$} & \multirow{2}{*}{$\mathrm{P} / \mathrm{L}$} & \multicolumn{3}{|c|}{$\mathrm{W}$} & \multirow{2}{*}{ Média de W } \\
\hline & & & Itaberá & Campinas & Maracaí & \\
\hline & $\mathrm{s}$ & $\mathrm{mmH}_{2} \mathrm{O} \cdot \mathrm{mm}^{-1}$ & 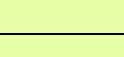 & $-\left(10^{-4} \mathrm{~J}\right)$ & $\overline{ }$ & \\
\hline ANAHUAC & 432 & 1,08 & 409 & 353 & 279 & 347 \\
\hline IAC 24 & 451 & 0,71 & 282 & 344 & 252 & 293 \\
\hline BR 18 & 432 & 0,62 & 346 & 286 & 231 & 288 \\
\hline IAPAR 28 & 411 & 1,14 & 305 & 307 & 218 & 277 \\
\hline IAC 332 & 463 & 0,63 & 366 & 253 & 190 & 270 \\
\hline IAC 350 & 330 & 0,91 & 299 & 294 & 187 & 260 \\
\hline IAC 289 & 356 & 0,86 & 303 & 300 & 161 & 255 \\
\hline IAPAR 60 & 512 & 0,95 & 288 & 240 & 141 & 223 \\
\hline IAC 120 & 367 & 0,55 & 241 & 209 & 205 & 218 \\
\hline OCEPAR 14 & 424 & 0,52 & 262 & 238 & 150 & 217 \\
\hline IAC 287 & 404 & 0,55 & 255 & 222 & 169 & 215 \\
\hline IAC 348 & 372 & 0,52 & 238 & 208 & 184 & 210 \\
\hline IAC 347 & 393 & 0,74 & 224 & 236 & 157 & 206 \\
\hline PANDA & 376 & 0,60 & 240 & 193 & 182 & 205 \\
\hline IAC 346 & 377 & 0,68 & 273 & 208 & 128 & 203 \\
\hline IAC 304 & 380 & 0,82 & 235 & 232 & 131 & 200 \\
\hline IAC 60 & 393 & 0,31 & 192 & 178 & 128 & 166 \\
\hline IAC 319 & 317 & 0,52 & 213 & 157 & 117 & 162 \\
\hline IAC 227 & 340 & 0,49 & 171 & 172 & 131 & 158 \\
\hline IAC 340 & 346 & 0,29 & 132 & 156 & 89 & 126 \\
\hline
\end{tabular}

favoráveis para a qualidade dos grãos. As farinhas provenientes dos genótipos Anahuac e IAPAR 28 apresentaram altos valores no índice de configuração da curva $(\mathrm{P} / \mathrm{L})$, indicativos de farinhas muito elásticas, e os genótipos IAC 340, IAC 60 e IAC 227, baixos valores no referido índice, indicativos de farinhas pouco elásticas. Segundo PYLER (1973), quanto maior a elasticidade de uma farinha, maior a sua força, ou seja, maior a quantidade de glutenina, proteína insolúvel formadora do glúten. Os valores de $\mathrm{W}$ obtidos, representados pela superfície da curva indicativa da força de panificação da farinha, com exceção dos genótipos IAC 340, IAC 227, IAC 319 e IAC 60, são indicativos de farinha de glúten forte (GERMANI e CARVALHO, 1998).

A caracterização das farinhas no farinógrafo quanto à capacidade de absorver água, indicativa da quantidade necessária para que a massa tenha uma consistência definida, e a avaliação do comportamento da massa durante o processo mecânico de amas- samento, demonstraram que as farinhas provenientes dos genótipos Anahuac, IAC 332, IAPAR 28, IAC 289, IAC 24, BR 18, IAC 350 e IAPAR 60 apresentaram características de farinha forte, com altos valores de absorção, estabilidade e valor volumétrico. Incluemse nesse grupo, 'IAC 348' e 'IAC 287', apesar de terem apresentado baixo índice de absorção, característica de trigos de grãos moles (Quadro 7). Os genótipos IAC 120, OCEPAR 14, Panda, IAC 304 e IAC347 apresentaram valores médios de absorção, índices de estabilidade entre 5,73 e 7,90 min (dados indicativos de farinha de média qualidade) e valores relativamente bons de VV. Os genótipos IAC 60, IAC 346, IAC 319, IAC 227 e IAC 340 acusaram baixos índices de estabilidade e de valor volumétrico e, dentre os mesmos, os genótipos IAC 60 e IAC 340 apresentaram ainda valores baixos de absorção, característica normal de farinha fraca.

Os resultados mostraram que as diferenças entre as regionais contribuíram para influenciar na 
Quadro 7. Parâmetros farinográficos como: estabilidade (EST) por local, médias de absorção de água (ABS) e valor volumétrico (VV), de genótipos de trigo, cultivados em Itaberá e em Maracaí, em condição de sequeiro e em Campinas com irrigação por aspersão, no biênio 1994-95

\begin{tabular}{|c|c|c|c|c|c|c|}
\hline \multirow[t]{2}{*}{ Genótipo } & & $\begin{array}{l}\text { Ita- } \\
\text { berá }\end{array}$ & $\begin{array}{l}\text { Cam- } \\
\text { pinas }\end{array}$ & $\begin{array}{c}\text { Mara- } \\
\text { caí }\end{array}$ & $\begin{array}{l}\text { Mé- } \\
\text { dia }\end{array}$ & \multirow[b]{2}{*}{ VV } \\
\hline & ABS & EST & EST & EST & EST & \\
\hline & $\%$ & \multicolumn{4}{|c|}{$\longrightarrow \min \longrightarrow$} & \\
\hline Anahuac & 58,94 & 26,25 & 21,15 & 8,05 & 18,48 & 74,66 \\
\hline IAC 348 & 51,86 & 21,00 & 13,70 & 8,30 & 14,33 & 70,33 \\
\hline IAC 287 & 53,66 & 24,04 & 10,85 & 5,60 & 13,50 & 63,00 \\
\hline IAC 332 & 59,24 & 21,30 & 7,00 & 5,70 & 11,33 & 64,00 \\
\hline IAPAR 28 & 60,43 & 14,85 & 11,30 & 6,50 & 10,88 & 69,33 \\
\hline IAC 289 & 58,33 & 13,40 & 12,30 & 5,80 & 10,50 & 65,00 \\
\hline IAC 24 & 61,46 & 11,05 & 13,00 & 6,70 & 10,25 & 66,16 \\
\hline BR 18 & 59,11 & 11,40 & 11,35 & 4,55 & 9,10 & 64,83 \\
\hline IAC 350 & 59,80 & 11,55 & 8,95 & 5,05 & 8,51 & 62,83 \\
\hline IAPAR 60 & 59,91 & 12,75 & 7,50 & 3,80 & 8,01 & 60,83 \\
\hline IAC 120 & 55,08 & 10,00 & 6,80 & 6,10 & 7,90 & 61,50 \\
\hline OCEPAR 14 & 58,60 & 9,15 & 9,95 & 3,75 & 7,61 & 60,50 \\
\hline Panda & 56,73 & 7,45 & 6,50 & 5,65 & 6,53 & 59,83 \\
\hline IAC 304 & 58,31 & 7,65 & 5,80 & 4,25 & 5,90 & 58,83 \\
\hline IAC 347 & 59,16 & 6,40 & 6,10 & 4,70 & 5,73 & 58,66 \\
\hline IAC 60 & 52,95 & 7,05 & 5,80 & 3,50 & 5,45 & 55,66 \\
\hline IAC 346 & 59,40 & 6,00 & 6,40 & 3,60 & 5,33 & 60,50 \\
\hline IAC 319 & 57,55 & 7,15 & 4,15 & 3,35 & 4,88 & 52,83 \\
\hline IAC 227 & 59,69 & 5,05 & 5,75 & 3,40 & 4,73 & 55,83 \\
\hline IAC 340 & 52,21 & 4,00 & 4,85 & 3,05 & 3,96 & 49,66 \\
\hline
\end{tabular}

qualidade tecnológica da farinha dos genótipos avaliados, conforme pode ser verificado nos dados relativos a W e EST, nos quadros 6 e 7. Em Maracaí (Zona A) os dados desses parâmetros para todos os genótipos foram inferiores aos obtidos em Itaberá (Zona B) e em Campinas (Zona H), e, dentro de cada local, as variações foram detectadas de acordo com as características genéticas de cada genótipo. Considerando que as precipitações pluviais de Maracaí foram inferiores às de Itaberá (figura 1) - região que se caracteriza por apresentar maiores índices pluviais no período de inverno, no Estado de São Paulo (KALCKMANn et al. 1965) - os resultados obtidos estão de acordo com Mazzoni e Robutti (1983), para os quais, a qualidade do glúten pode ser influenciada por altas temperaturas e baixa umidade relativa do ar durante o período de maturação do trigo.

\section{CONCLUSÕES}

1. Os genótipos mostraram comportamento regional diferenciado quanto ao rendimento de grãos, com interação significativa entre ano vs. local nas condições de sequeiro, ocorrendo diferenças acentuadas no rendimento de grãos entre as Zonas A e B;

2. Os genótipos IAC 289, IAC 340 e BR 18 mostraram, na média, maior rendimento de grãos; 'IAC 289', 'IAC 340' e 'IAC 304' apresentaram-se instáveis, de acordo com as condições do ambiente, sendo responsivos em ambientes favoráveis; e 'IAC 227', adaptado em ambientes desfavoráveis;

3. Os genótipos apresentaram variação quanto à tolerância à toxicidade de alumínio, sendo 'Anahuac', 'IAC 340', 'IAC 287' e 'IAC 304' muito sensíveis e 'IAC 24', 'IAC 120', 'IAC 227', 'OCEPAR 14', 'IAC 348', 'Panda', 'BR 18', 'IAC 319', 'IAPAR 60', 'IAC 332' e 'IAC 347', tolerantes;

4. Os genótipos de textura dura apresentaram maior extração de farinha em relação aos de textura mole. Estes, de maneira geral, apresentaram farinhas com baixa atividade amilásica;

5. Os genótipos IAC 340, IAC 227, IAC 319 e IAC 60 apresentaram farinhas com baixos índices do valor de W (energia de deformação da massa) e baixa estabilidade, características de farinha de glúten fraco. Os demais genótipos revelaram característica de glúten forte;

6. Os trigos semeados em condições de sequeiro, em Itaberá, apresentaram melhor qualidade tecnológica do que aqueles semeados em Maracaí.

\section{REFERÊNCIAS BIBLIOGRÁFICAS}

AMERICAN ASSOCIATION OF CEREAL CHEMISTS (AACC). Approved methods of the American Association of Cereal Chemists. 8 ed. St. Paul, 1983. v.1.

BRASIL. Instruções Normativas n.1, de 27/01/99. Ministério da Agricultura e do Abastecimento. Diário Oficial da União, Brasília, seção 1, p.132, 1999.

CAMARGO, C.E.O.; OLIVEIRA, O.F. Tolerância de cultivares de trigo a diferentes níveis de alumínio em solução nutritiva e no solo. Bragantia, Campinas, v.40, n.1, p.21-31, 1981.

CAMARGO, C.E.O.; FELICIO, J.C.; ROCHA JUNIOR, L.S. Trigo: tolerância ao alumínio em solução nutritiva. Bragantia, Campinas, v.46, n.2, p.183-190, 1987.

CAMPINAS. Instituto Agronômico. Reunião da Comissão Técnica de Trigo da Secretaria de Agricultura e Abastecimento do Estado de São Paulo: recomendações para 1996. Campinas, 1996. 68p. (Boletim Técnico, 167) 
CARVALHO, F.I.F. de. Genética quantitativa. In: FUNDAÇÃO CARGILL. Trigo no Brasil. Campinas, 1982. v.1, p.65-94.

DUARTE, A.R. Tecnologia na agricultura: considerações sobre a produtividade do trigo brasileiro. São Paulo, 1974. 144p. Tese (Doutorado) - USP.

EBERHART, S.A.; RUSSELL, W.A. Stability parameters for comparing varieties. Crop Science, Madison, v.6, p.3640, 1966.

GERMANI, R.; CARVALHO, J.L.V. de. Perfil tecnológico de cultivares de trigo. Rio de Janeiro: EMBRAPA-CTAA, 1998. 72p. (EMBRAPA-CTAA. Documentos, n.32).

HANSON, H.; BORLAUG, N.E.; ANDERSON, R.G. Trigo en el tercer mundo. Mexico: Centro Internacional de Mejoramiento de Maiz y Trigo, 1982. 166p.

KALCKMANN, R.E.; ARRUDA, A.A.G.; HOETGEBAUM, F.; POPA, W.; BALDANZI, G.; GODOY, L.C. de. Regiões de trigo no Brasil: $1^{a}$ aproximação agrícola. Pelotas: IBGE, 1965. 104p. (IBGE. Estudos Técnicos, 28)

MANDARINO, J.M.G. Aspectos importantes para a qualidade do trigo. Londrina: EMBRAPA-CNPSo, 1993, 32p. (Documento, 60)

MAZZONI, M.R.C.; ROBUTTI, J.L. Evaluación del método de sedimentación "Dodecil Sulfato de Sodio" para determinar calidad panadera em mejoramiento de trigo. Buenos Aires:
Instituto Nacional de Tecnología Agropecuaria INTA, 1983. 12p. (Informe Técnico, 185)

MOTA, F.S. da. Clima e Zoneamento para a triticultura no Brasil. In: FUNDAÇÃO CARGILL. Trigo no Brasil. Campinas, 1982. v.1, p.27-61.

MOORE, D.P.; KRONSTAD, W.E.; METZGER, R.J. Screening wheat for aluminum tolerance. In: WORKSHOP ON PLANT ADAPTATIONS TO MINERAL STRESS IN PROBLEM SOILS, Beltsville, Maryland. 1976. Proceedings. Ithaca, Cornell University, 1976. p.287-295.

PIMENTEL-GOMES, F. Curso de Estatística Experimental. Piracicaba: ESALQ/USP, 1970. 348p.

PYLER, E.L. Baking Science E Technology. Chicago, Siebel Publishing, 1973. v.1, chap.3, p.83-119; chap.7, p.236336.

RAIJ, B. van; CANTARELLA, H; QUAGGIO, J.A.; FURLANI, A.M.C. Recomendações de adubação e calagem para o Estado de São Paulo. Campinas: Instituto Agronômico Fundação IAC, 1996. 285p. (Boletim T écnico, 100)

SILVA, E.M.; LUCHIARI JUNIOR, A.; GUERRA, A.F.; GOMIDE, R.L. Recomendações sobre o manejo de irrigação em trigo para a região dos cerrados. In: REUNIÃO DA COMISSÃO NORTE-BRASILEIRA DE PESQUISA DE TRIGO, 10., Campinas, 1984. Ata. Brasília, EMBRAPACPAC, 1984.60p. 